\title{
Paecilomyces keratitis with corneal perforation salvaged by a conjunctival flap and delayed keratoplasty
}

\author{
Satoshi Mizunoya, Yoshihiro Watanabe
}

Early diagnosis of fungal keratitis is difficult, and it has been generally associated with poor visual prognosis. We treated a man who had keratomycosis with corneal perforation and lens prolapse caused by Paecilomyces sp, which rarely causes ocular infection. The patient was given antifungal agents, a temporary conjunctival flap, and penetrating keratoplasty. His visual outcome was successful.

\section{Case report}

On 7 October 1991, a 72-year-old man who had a 23-year history of diabetes was referred to our clinic with keratitis in his left eye. He was diagnosed as having iridocyclitis and had been treated with topical steroids and antibiotics at another clinic for several months. His left visual acuity was hand motion, and slit-lamp examination of the left eye disclosed a severely injected

Department of Ophthalmology, Chiba University School of Medicine, Japan S Mizunoya

\section{Department of} Ophthalmology, Narita Red Cross Hospital, Japan

YWatanabe

Correspondence to: Satoshi Mizunoya, MD, Department of

Ophthalmology, Chiba

University School of Medicine

1-8-1 Inohana, Chuo-ku, Chiba 260, Japan.

Accepted for publication 26 August 1993 conjunctiva and central corneal ulcer. The ulcer was $6 \mathrm{~mm}$ in diameter and its margin was blurred. The lesion was whitish and the surface was somewhat elevated. There was no satellite lesion or hypopyon (Fig 1A).

Treatment with natamycin drops and intravenous miconazole ( $400 \mathrm{mg}$ per day) was started because fungal elements were identified from the smear of the legion. Paecilomyces sp appeared on Sabouraud glucose agar after 5 days of culture (Fig 1B). The corneal infiltration absorbed gradually, but a large corneal perforation occurred 16 days after the treatment was started (Fig 1C).

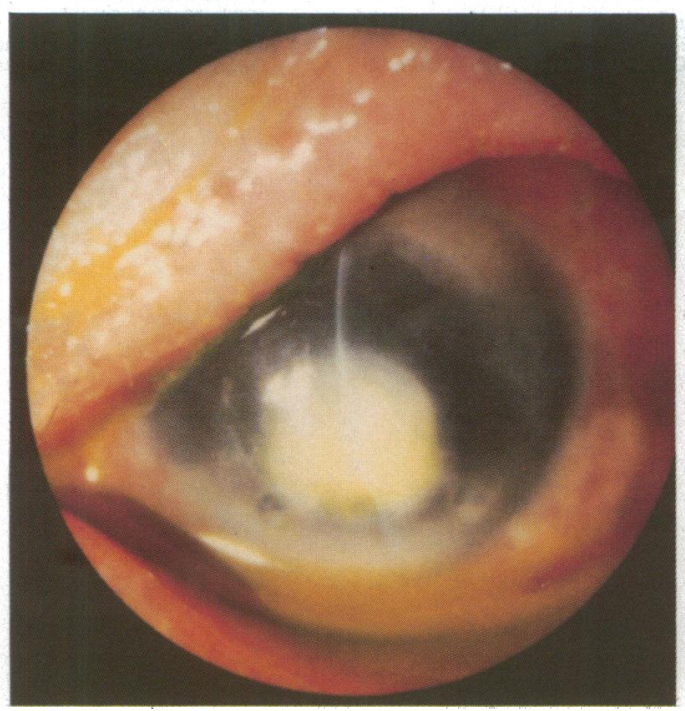

Figure 1A At the initial examination, the left cornea demonstrated a lesion $6 \mathrm{~mm}$ in diameter. The round and slightly elevated white infiltration had an irregular border.

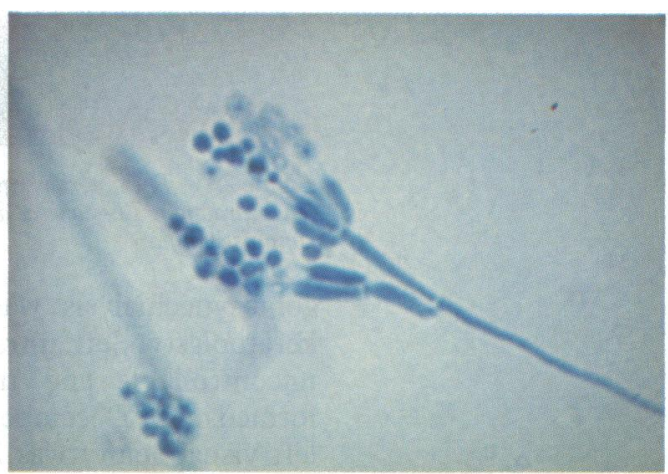

Figure 1B Hyphae, branching conidiophores with whorls of phialides and chains of conidia characterise Paecilomyces $s p$ in the slide culture at 5 days. Phialides arising from the hyphae themselves is also a characteristic of this species (lactophenol cotton blue, $\times 75$ ).

An intracapsular lens extraction was performed immediately through the central perforated corneal lesion, and the defect was covered by an inferior bulbar conjunctival flap temporarily (Fig 2A). The antifungal therapy was continued for 10 days after the surgery and the total dose of miconazole was $9600 \mathrm{mg}$ for 24 days. Fourteen days after the operation, the inflammatory symptoms of conjunctival injection and corneal vascularisation were improved. On 9 November 1991, left penetrating keratoplasty was performed. When the conjunctival flap was removed, a nearly total peripheral anterior synechiae of the iris was noted, and

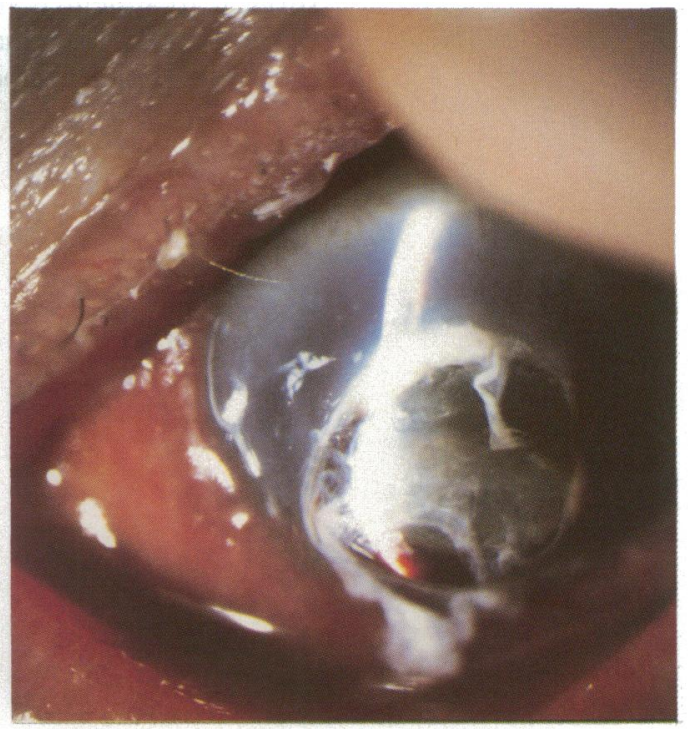

Figure IC The anterior segment 16 days after admission shows the lesion with a corneal defect $6 \mathrm{~mm}$ in diameter. The lens and iris had prolapsed from the defect. 


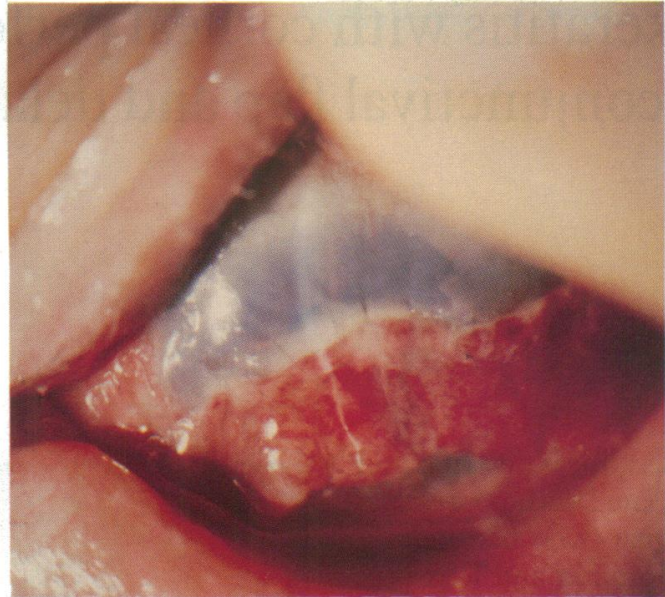

Figure $2 A$ The anterior segment the day after intracapsular lens extraction. The cormeal defect was covered by a conjunctival flap.

goniosynechialysis was impossible. After the keratoplasty, left intraocular pressure became uncontrollable and trabeculectomy was performed on 25 December 1991. One year later, his left visual acuity was 20/80, and his left intraocular pressure was maintained within normal limits without medication (Fig 2B).

\section{Comment}

Paecilomyces $\mathrm{sp}$ is a common fungus but only a few cases of ocular infection caused by this species have been reported. ${ }^{1-4}$ Most patients had a poor visual prognosis.

We treated our patient topically with natamycin and systemically with miconazole. Natamycin has a wide spectrum of coverage, but it is often ineffective for deep corneal lesions because of its poor penetration into the cornea. ${ }^{5}$ Miconazole is one of the imidazole antifungal agents. It retains a high concentration in blood and has good penetration into ocular tissues when administered intravenously. ${ }^{6}$ Although we believe our medication was effective, advanced stromal destruction resulted in corneal perforation.

When a large corneal perforation occurs, surgical treatment is inevitable. Primary penetrating keratoplasty may be an ideal therapy if the corneal lesion can be completely removed. In

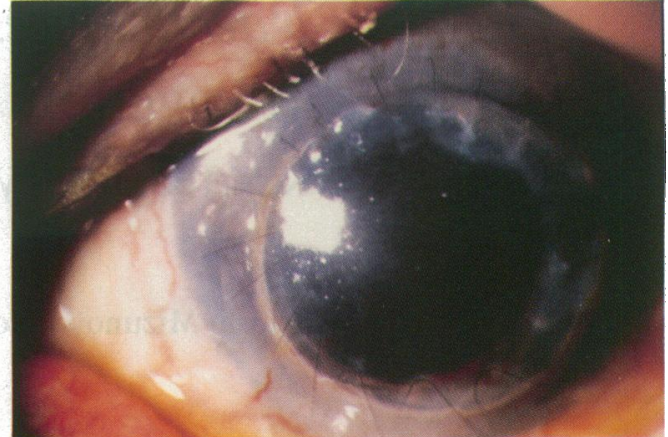

Figure $2 B$ The anterior segment at 3 months after the patient was hospitalised shows no inflammation and a clear graft. Left visual acuity had recovered to $20 / 80$ from hand motion.

our patient, however, the lesion extended close to the inferior limbus, and to circumscribe the lesion with a safe margin would have been difficult. We thought that the narrow host rim would result in postoperative vessel growth to the sutures and graft rejection. For those reasons, we selected the conjunctival flap, which reportedly provides a direct route for a cell mediated immune response and promotes the healing of corneal mycosis. We believe that in our patient the conjunctival flap reduced the danger of recurrent infection and graft rejection. On the other hand, covering the large corneal defect with the conjunctival flap produced the anterior chamber shallowing, wide peripheral anterior synechiae, and secondary glaucoma postoperatively. Therefore, when and how to treat keratomycosis with large perforation is still a matter for consideration.

We are grateful to Professor E Adachi-Usami for her valuable advice.

1 Minogue MJ, Francis IC, Quatermass P, Kappagoda MB Bradbury R, Walls RS, et al. Successful treatment of funga keratitis caused by Paecilomyces lilacinus. Am $\mathcal{f}$ Ophthalmo 1984; 98: 626-7.

2 Gordon MA, Norton SW. Corneal transplant infection by Paecilomyces lilacinus. Sabouraudia 1985; 23: 295-301.

3 Starr MB. Paecilomyces lilacinus keratitis: two case reports in extended wear contact lens wearers. $C L A O \mathcal{F} 1987$; 13: 95 101

4 Minogue MJ, Playfair TJ, Gregory-Roberts JC, Robinson LP. Cure of Paecilomyces endophthalmitis with multiple intra Cure of Paecilomyces endophthalmitis with multiple intravitreal injections of amph

5 Jones BR. Principles in the management of oculomycosis. XXXI Edward Jackson Memorial Lecture. Am $\mathcal{F}$ Ophthalmo 1975; 79: 719-51.

6 Foster CS. Miconazole therapy for keratomycosis. Am $\mathcal{J}$ Ophthalmol 1981; 91: 622-9. 